\title{
A Hölder type inequality and an interpolation theorem in Euclidean Jordan algebras
}

\author{
M. Seetharama Gowda \\ Department of Mathematics and Statistics \\ University of Maryland, Baltimore County \\ Baltimore, Maryland 21250, USA \\ gowda@umbc.edu
}

September 17, 2018

\begin{abstract}
In a Euclidean Jordan algebra $\mathcal{V}$ of rank $n$ which carries the trace inner product, to each element $x$ we associate the eigenvalue vector $\lambda(x)$ whose components are the eigenvalues of $x$ written in the decreasing order. For any $p \in[1, \infty]$, we define the spectral $p$-norm of $x$ to be the $p$-norm of $\lambda(x)$ in $\mathcal{R}^{n}$. In this paper, we show that $\|x \circ y\|_{1} \leq\|x\|\left\|_{p}\right\| y \|_{q}$, where $x \circ y$ denotes the Jordan product of two elements $x$ and $y$ in $\mathcal{V}$ and $q$ is the conjugate of $p$. For a linear transformation on $\mathcal{V}$, we state and prove an interpolation theorem relative to these spectral norms. In addition, we compute/estimate the norms of Lyapunov transformations, quadratic representations, and positive transformations on $\mathcal{V}$.
\end{abstract}

Key Words: Euclidean Jordan algebra, Hölder type inequality, strong operator commutativity, majorization, Schur-convexity, positive transformation, interpolation theorem.

AMS Subject Classification: 15A18, 15A60, 17C20 


\section{Introduction}

The classical Hölder and Minkowski inequalities, when stated in the setting of $\mathcal{R}^{n}$, say that for two real vectors $x=\left(x_{1}, x_{2}, \ldots, x_{n}\right)$ and $y=\left(y_{1}, y_{2}, \ldots, y_{n}\right)$ and for any $p \in[1, \infty]$ with conjugate $q$ (that is, $p^{-1}+q^{-1}=1$ ),

$$
\left|\sum_{1}^{n} x_{i} y_{i}\right| \leq \sum_{1}^{n}\left|x_{i} y_{i}\right| \leq\|x\|_{p}\|y\|_{q} \quad \text { and } \quad\|x+y\|_{p} \leq\|x\|_{p}+\|y\|_{p},
$$

where $\|x\|_{p}$ denotes the $p$-norm of $x$, etc. Viewing $\mathcal{R}^{n}$ as a Euclidean Jordan algebra with Jordan product $x \circ y:=\left(x_{1} y_{1}, x_{2} y_{2}, \ldots, x_{n} y_{n}\right)$, inner product $\langle x, y\rangle=\sum_{1}^{n} x_{i} y_{i}$, and components of $x$ as eigenvalues of $x$, we may restate the above inequalities as

$$
|\langle x, y\rangle| \leq\|\lambda(x \circ y)\|_{1} \leq\|\lambda(x)\|_{p}\|\lambda(y)\|_{q} \quad \text { and } \quad\|\lambda(x+y)\|_{p} \leq\|\lambda(x)\|_{p}+\|\lambda(y)\|_{p},
$$

where $\lambda(x)$ denotes the vector of eigenvalues (here, entries) of $x$ written in the decreasing order, etc. Motivated by the appearance of inequalities of the above type in various matrix theory settings (especially for real symmetric or complex Hermitian matrices) and in the optimization literature over symmetric cones, we raise the issue of proving such inequalities over general Euclidean Jordan algebras. To elaborate, let $(\mathcal{V}, \circ,\langle\cdot, \cdot\rangle)$ be a Euclidean Jordan algebra of rank $n$ [5], [8]. We assume that $\mathcal{V}$ carries the trace inner product, that is, $\langle x, y\rangle:=\operatorname{tr}(x \circ y)$. For each $x \in \mathcal{V}$, we associate the eigenvalue vector $\lambda(x)$ in $\mathcal{R}^{n}$ whose entries are the eigenvalues of $x$ written in the decreasing order. For $p \in[1, \infty]$, we define the spectral $p$-norm on $\mathcal{V}$ by

$$
\|x\|_{p}:=\|\lambda(x)\|_{p}
$$

where the right-hand side denotes the $p$-norm of the vector $\lambda(x)$ in $\mathcal{R}^{n}$. Using majorization ideas, a generalization of Thompson's triangle inequality, and case-by-case analysis (of five types of simple Euclidean Jordan algebras), Tao et al., [20] have shown that $\|\cdot\|_{p}$ is a norm on $\mathcal{V}$ thereby establishing the Minkowski inequality in the setting of Euclidean Jordan algebras. For a comprehensive proof based on majorization and Schur-convexity theorem, see [13]. Regarding the Hölder inequality, Tao et al. [20] have also shown that the inequality $|\langle x, y\rangle| \leq\|x\|_{p}\|y\|_{q}$ holds for all $x$ and $y$ when $\mathcal{V}$ is a simple Euclidean Jordan algebra. For $p=2$, the inequality $\|x \circ y\|_{1} \leq\|x\|_{2}\|y\|_{2}$ was proved in [23] and [17]. Going beyond these special cases, in this paper we establish the inequalities

$$
|\langle x, y\rangle| \leq\|x \circ y\|_{1} \leq\|x\|_{p}\|y\|_{q}
$$

over general Euclidean Jordan algebras. Our related contributions include an interpolation theorem for linear transformations on $\mathcal{V}$ relative to the spectral norms and computation/estimation of norms of Lyapunov transformations, quadratic representations, and positive transformations.

In the first part of our paper, we establish the following Hölder type inequality. To explain, we introduce a notation and a definition. Given any Jordan frame $\left\{e_{1}, e_{2}, \ldots, e_{n}\right\}$ in $\mathcal{V}$, we consider 
the ordered Jordan frame $\mathcal{E}:=\left(e_{1}, e_{2}, \ldots, e_{n}\right)$ and write, for any $x \in \mathcal{V}$,

$$
\lambda(x) * \mathcal{E}:=\sum_{1}^{n} \lambda_{i}(x) e_{i} .
$$

We say that two elements $x$ and $y$ in $\mathcal{V}$ strongly operator commute if there is an ordered Jordan frame $\mathcal{E}$ such that $x=\lambda(x) * \mathcal{E}$ and $y=\lambda(y) * \mathcal{E}$.

Theorem 1.1 (A Hölder type inequality in Euclidean Jordan algebras) Let $x, y \in \mathcal{V}$ and $p \in[1, \infty]$ with conjugate $q$. Then,

$$
\|x \circ y\|_{1} \leq\|x\|_{p}\|y\|_{q}
$$

Moreover, equality holds in (10) if and only if

(a) $x$ and $y \circ \varepsilon$ strongly operator commute and

(b) $\langle\lambda(x), \lambda(y \circ \varepsilon)\rangle=\|\lambda(x)\|_{p}\|\lambda(y)\|_{q}$ holds in $\mathcal{R}^{n}$,

where $x \circ y$ has the spectral decomposition $x \circ y=\left(z_{1} e_{1}+z_{2} e_{2}+\cdots+z_{k} e_{k}\right)-\left(z_{k+1} e_{k+1}+\cdots+z_{n} e_{n}\right)$ for some $k, 0 \leq k \leq n$ and $z_{i} \geq 0$ for all $i$, and $\varepsilon:=\left(e_{1}+e_{2}+\cdots+e_{k}\right)-\left(e_{k+1}+\cdots+e_{n}\right)$.

Our proof of the above result is based on the following generalization of the Fan-Theobald trace inequality of matrix theory (which is related to von Neumann's trace inequality). The inequality (2) given below extends the so-called rearrangement inequality of Hardy, Littlewood, and Pólya when $\mathcal{V}=\mathcal{R}^{n}$ [16] and the Fan-Theobald trace inequality [4], 22] when $\mathcal{V}=\mathcal{S}^{n}$ or $\mathcal{H}^{n}$ (the algebras of $n \times n$ real/complex Hermitian matrices). For simple Euclidean Jordan algebras, this result has been observed in [14, [10]. Based on this simple algebra result, the rearrangement inequality, and the fact that any Euclidean Jordan algebra is a product of simple algebras, one can prove the general result. For a different and comprehensive proof, see [1].

Theorem 1.2 (A generalized Fan-Theobald trace inequality) Let $x, y \in \mathcal{V}$. Then,

$$
\langle x, y\rangle \leq\langle\lambda(x), \lambda(y)\rangle .
$$

Moreover, equality holds in (2) if and only if $x$ and $y$ strongly operator commute.

In the second part of the paper, we compute/estimate the (spectral) norms of the Lyapunov transformation $L_{a}$ defined by $L_{a}(x):=a \circ x$, the quadratic representation $P_{a}$ defined by $P_{a}:=2 L_{a}^{2}-L_{a^{2}}$, and a positive (linear) transformation defined by the condition $x \geq 0 \Rightarrow P(x) \geq 0$.

In the final part of the paper, we describe an interpolation theorem for a linear transformation on $\mathcal{V}$ relative to the spectral norms. Based on the $K$-method of real interpolation theory [15], we 
show that

$$
\|T\|_{p \rightarrow p} \leq\|T\|_{r \rightarrow r}^{1-\theta}\|T\|_{s \rightarrow s}^{\theta}
$$

where $T: \mathcal{V} \rightarrow \mathcal{V}$ is a linear transformation with $\|T\|_{p \rightarrow p}$ denoting the the norm of $T$ relative to the spectral $p$-norm and real numbers $r, s, p \in[1, \infty]$ are related by $\frac{1}{p}=\frac{1-\theta}{r}+\frac{\theta}{s}$ for some $\theta \in[0,1]$.

\section{Preliminaries}

The symbol $\mathcal{R}^{n}$ denotes the usual Euclidean $n$-space in which we regard elements as either row vectors or column vectors depending on the context. Throughout this paper, $(\mathcal{V}, \circ,\langle\cdot, \cdot\rangle)$ denotes a Euclidean Jordan algebra of rank $n$ and unit element $e$ [5], [8], with $x \circ y$ denoting the Jordan product and $\langle x, y\rangle$ denoting the inner product of $x$ and $y$ in $\mathcal{V}$. We specifically note that

$$
\langle x \circ y, z\rangle=\langle x, y \circ z\rangle \quad \text { for all } x, y, z \in \mathcal{V} .
$$

For convenience, we use the same inner product notation in $\mathcal{R}^{n}$ (which carries the usual inner product) and in $\mathcal{V}$.

It is known that any Euclidean Jordan algebra is a direct product/sum of simple Euclidean Jordan algebras and every simple Euclidean Jordan algebra is isomorphic one of five algebras, three of which are the algebras of $n \times n \mathrm{real} /$ complex/quaternion Hermitian matrices. The other two are: the algebra of $3 \times 3$ octonion Hermitian matrices and the Jordan spin algebra. We let $\mathcal{S}^{n}\left(\mathcal{H}^{n}\right)$ denote the algebra of all $n \times n$ real symmetric (respectively, complex Hermitian) matrices.

According to the spectral decomposition theorem [5], any element $x \in \mathcal{V}$ has a decomposition

$$
x=x_{1} e_{1}+x_{2} e_{2}+\cdots+x_{n} e_{n},
$$

where the real numbers $x_{1}, x_{2}, \ldots, x_{n}$ are (called) the eigenvalues of $x$ and $\left\{e_{1}, e_{2}, \ldots, e_{n}\right\}$ is a Jordan frame in $\mathcal{V}$. (An element may have spectral decompositions coming from different Jordan frames, but the eigenvalues remain the same.) Then, $\lambda(x)$ - called the eigenvalue vector of $x$ - is the vector of eigenvalues of $x$ written in the decreasing order. The trace and spectral p-norm of $x$ are defined by

$$
\operatorname{tr}(x):=x_{1}+x_{2}+\cdots+x_{n} \quad \text { and } \quad\|x\|_{p}:=\|\lambda(x)\|_{p},
$$

where $\|\lambda(x)\|_{p}$ denotes the usual $p$-norm of the vector $\lambda(x)$ in $\mathcal{R}^{n}$. (Note that $\|x\|_{p}$ is the $p$-norm of any vector in $\mathcal{R}^{n}$ formed by $x_{1}, x_{2}, \ldots, x_{n}$.)

We use the notation $x \geq 0(x \leq 0, x>0)$ when all the eigenvalues of $x$ are nonnegative (respectively, nonpositive, positive). Also, $x \geq y$ (or $y \leq x$ ) means that $x-y \geq 0$. When $x \geq 0$ and has the spectral decomposition $x=\sum x_{i} e_{i}$, we define $\sqrt{x}:=\sum \sqrt{x_{i}} e_{i}$.

An element $c$ in $\mathcal{V}$ is an idempotent if $c^{2}=c$. Corresponding to such an element, the Peirce 
decomposition of $\mathcal{V}$ is the orthogonal direct sum ([5], page 62 and Proposition IV.1.1)

$$
\mathcal{V}=\mathcal{V}(c, 1) \oplus \mathcal{V}\left(c, \frac{1}{2}\right) \oplus \mathcal{V}(c, 0)
$$

where $\mathcal{V}(c, \gamma):=\{x \in \mathcal{V}: x \circ c=\gamma x\}$ and $\gamma \in\left\{0, \frac{1}{2}, 1\right\}$. Here $\mathcal{V}(c, 1)$ and $\mathcal{V}(c, 0)$ are subalgebras of $\mathcal{V}$ and $\mathcal{V}(c, 1) \circ \mathcal{V}(c, 0)=\{0\}$. There is another related Peirce decomposition of $\mathcal{V}$ : Corresponding to a Jordan frame $\left\{e_{1}, e_{2}, \ldots, e_{n}\right\}$, let $\mathcal{V}_{i i}:=\mathcal{V}\left(c_{i}, 1\right)=\mathcal{R} e_{i}$ and for $i \neq j, \mathcal{V}_{i j}:=\mathcal{V}\left(c_{i}, \frac{1}{2}\right) \cap \mathcal{V}\left(c_{j}, \frac{1}{2}\right)$. Then $\mathcal{V}$ is the orthogonal direct sum of subspaces $\mathcal{V}_{i j}$ ([5], Theorem IV.2.1). Hence any element $x \in \mathcal{V}$ has a Peirce decomposition: $x=\sum_{i \leq j} x_{i j}$, where $x_{i j} \in \mathcal{V}_{i j}$.

Now, starting from the given inner product in $\mathcal{V}$, one can define the trace inner product $\langle x, y\rangle_{t r}:=$ $\operatorname{tr}(x \circ y)$ on $\mathcal{V}$ which is also compatible with the given Jordan product ([5], Prop. II.4.3 and Prop. III.1.5). Various concepts/results/decompositions remain the same when the given inner product is replaced by the trace inner product; in particular, for an element in $\mathcal{V}$, the spectral decomposition, eigenvalues, and trace remain the same. Under this trace inner product, the norm of any primitive element (such as an element in a Jordan frame) is one and so every Jordan frame becomes an orthonormal set. From now on, throughout this paper, we assume that the inner product is the trace inner product, that is, $\langle x, y\rangle=\operatorname{tr}(x \circ y)$.

Given a spectral decomposition $a=\sum a_{i} e_{i}$, we write

$$
|a|:=\sum\left|a_{i}\right| e_{i} \quad \text { and } \quad\|a\|_{1}=\sum\left|a_{i}\right|=\operatorname{tr}(|a|)
$$

With this notation, we observe that

$$
|\langle x, y\rangle|=|\operatorname{tr}(x \circ y)| \leq \operatorname{tr}(|x \circ y|)=\|x \circ y\|_{1} .
$$

Recall that two elements $x$ and $y$ in $\mathcal{V}$ strongly operator commute if there is an ordered Jordan frame $\mathcal{E}$ such that $x=\lambda(x) * \mathcal{E}$ and $y=\lambda(y) * \mathcal{E}$. The terms 'simultaneous order diagonalization' and 'similar joint decomposition' have also been used in the literature [14, [1]. Note that this notion is stronger than the usual operator commutativity where it is required that $x$ and $y$ have their spectral decompositions with respect to a common Jordan frame (or equivalently, the linear operators $L_{x}$ and $L_{y}$ commute, where $L_{x}(z):=x \circ z$, etc.) For example, in $R^{2}$, the vectors $x=(1,0)$ and $y=(0,1)$ operator commute, but not strongly.

Given two (column) vectors $p$ and $q$ in $\mathcal{R}^{n}$, we say that $p$ is majorized by $q$ and write $p \prec q$ if $p=A q$ for some doubly stochastic matrix $A \in \mathcal{R}^{n \times n}[16]$. (So, $A$ is a nonnegative matrix with every row and column sum one. By a well-known result of Birkhoff, a doubly stochastic matrix is a convex combination of permutation matrices, see [3].) For $x, y \in \mathcal{V}$, we say that $x$ is majorized by $y$ and write $x \prec y$ if $\lambda(x) \prec \lambda(y)$ in $\mathcal{R}^{n}$. If $f: \mathcal{R}^{n} \rightarrow \mathcal{R}$ is a convex function and $F:=f \circ \lambda$, 
then (by the classical Schur-convexity theorem [16]), we have:

$$
x \prec y \Rightarrow F(x) \leq F(y) .
$$

See [13] for applications of this in Euclidean Jordan algebras.

Throughout this paper, for a real number $\alpha$, we let $\operatorname{sgn} \alpha$ denote 1,0 , or -1 according as whether $\alpha$ is positive, zero, or negative.

\section{Proof of Theorem 1.1}

Toward establishing (11), we first prove a weaker inequality given below. It is a consequence of Theorem 1.2 ,

Proposition 3.1 Let $x, y \in \mathcal{V}$ and $p \in[1, \infty]$ with conjugate $q$. Then,

$$
|\langle x, y\rangle| \leq\|x\|_{p}\|y\|_{q} .
$$

Equality holds in (6) if and only if, with $\eta:=\operatorname{sgn}\langle x, y\rangle$,

(i) $\eta x$ and $y$ strongly operator commute and

(ii) $\langle\lambda(\eta x), \lambda(y)\rangle=\|\lambda(\eta x)\|_{p}\|\lambda(y)\|_{q}$ holds in $\mathcal{R}^{n}$.

The inequality (6) for a simple algebra is noted in [20], Theorem 4.2. A partial result for the equality in a simple algebra is stated in [20], Corollary 4.2, where it is assumed that $x, y \geq 0$.

Proof. Without loss of generality, let $\langle x, y\rangle \neq 0$. Since $\eta:=\operatorname{sgn}\langle x, y\rangle$ (which is 1 or -1 ), from Theorem 1.2 and the (classical) Hölder's inequality in $\mathcal{R}^{n}$, we have

$$
|\langle x, y\rangle|=\langle\eta x, y\rangle \leq\langle\lambda(\eta x), \lambda(y)\rangle \leq\|\lambda(\eta x)\|_{p}\|\lambda(y)\|_{q}=\|x\|_{p}\|y\|_{q},
$$

where we note that $\|\lambda(\eta x)\|_{p}=\|x\|_{p}$. This proves the inequality (6). Suppose $|\langle x, y\rangle|=\|x\|_{p}\|y\|_{q}$. Then, from (7), $\langle\eta x, y\rangle=\langle\lambda(\eta x), \lambda(y)\rangle$. It follows from Theorem 1.2 that $\eta x$ and $y$ strongly operator commute. Also, from (7), we get the equality stated in (ii).

Now suppose conditions $(i)$ and $(i i)$ hold. Then, by an application of Theorem 1.2 and $(i i)$ we see that the inequalities in (7) turn into equalities.

We now come to the proof of Theorem 1.1, For the case of $\mathcal{V}=\mathcal{S}^{n}$ (or $\mathcal{H}^{n}$ ), the inequality (11) can be proved using known singular values inequalities: For an $n \times n$ real/complex matrix $A$, let $\sigma(A):=\left(\sigma_{1}(A), \sigma_{2}(A), \ldots, \sigma_{n}(A)\right)$ denote the vector of singular values of $A$ ( $=$ the eigenvalues of $\sqrt{A^{*} A}$ ) written in the decreasing order. Then, for any two matrices $A$ and $B$, we have the 
inequalities $\sum_{1}^{n} \sigma_{i}(A B) \leq\langle\sigma(A), \sigma(B)\rangle$ and $\sum_{1}^{n} \sigma_{i}(A+B) \leq \sum_{1}^{n} \sigma_{i}(A)+\sum_{1}^{n} \sigma_{i}(B)$ (see [12], Theorem 3.3.14 and Corollary 3.4.3). Using these, for any $X, Y \in \mathcal{S}^{n}$ or $\mathcal{H}^{n}$, we see that

$$
\begin{gathered}
\|X \circ Y\|_{1}:=\sum_{1}^{n}\left|\lambda_{i}(X \circ Y)\right|=\sum_{1}^{n} \sigma_{i}(X \circ Y)=\sum_{1}^{n} \frac{1}{2} \sigma_{i}(X Y+Y X) \\
\leq \frac{1}{2}\left(\sum_{1}^{n} \sigma_{i}(X Y)+\sum_{1}^{n} \sigma_{i}(Y X)\right) \leq\langle\sigma(X), \sigma(Y)\rangle \leq\|\sigma(X)\|_{p}\|\sigma(Y)\|_{q}=\|\lambda(X)\|_{p}\|\lambda(Y)\|_{q} .
\end{gathered}
$$

The proof given below, based on majorization techniques, is comprehensive and avoids looking at particular cases of simple algebras.

Proof. We fix $x, y \in \mathcal{V}$. If $x \circ y \geq 0$ or $x \circ y \leq 0$, then,

$$
\|x \circ y\|_{1}=\operatorname{tr}(|x \circ y|)=|\operatorname{tr}(x \circ y)|=|\langle x, y\rangle| \leq\|x\|_{p}\|y\|_{p}
$$

by Proposition 3.1. Moving away from these two cases, consider the spectral decomposition of $x \circ y$ which can be written in the following form: For some natural number $k, 1 \leq k<n$,

$$
x \circ y=\left(z_{1} e_{1}+z_{2} e_{2}+\cdots+z_{k} e_{k}\right)-\left(z_{k+1} e_{k+1}+\cdots+z_{n} e_{n}\right),
$$

where $z_{i} \geq 0$ for all $i$. Now, let $\varepsilon:=\left(e_{1}+e_{2}+\cdots+e_{k}\right)-\left(e_{k+1}+\cdots+e_{n}\right)$. Then, $\varepsilon^{2}=e$ and $|x \circ y|=(x \circ y) \circ \varepsilon$. We now claim that

$$
\|y \circ \varepsilon\|_{q} \leq\|y\|_{q}
$$

To see this, let $c=e_{1}+e_{2}+\cdots+e_{k}$. By the Peirce decomposition theorem [5], $\mathcal{V}$ is the orthogonal direct sum of $\mathcal{V}(c, 1), \mathcal{V}\left(c, \frac{1}{2}\right)$, and $\mathcal{V}(c, 0)$. Hence, we can write $y=u+v+w$, where $u \in \mathcal{V}(c, 1)$, $v \in \mathcal{V}\left(c, \frac{1}{2}\right)$, and $w \in \mathcal{V}(c, 0)$. Since $\varepsilon=2 c-e$, an easy computation shows that

$$
y \circ \varepsilon=u-w .
$$

As $\mathcal{V}(c, 1) \circ \mathcal{V}(c, 0)=\{0\}$, by working with the spectral decompositions of $u$ in $\mathcal{V}(c, 1)$ (which is a Euclidean Jordan algebra of rank $k$ ) and $w$ in $\mathcal{V}(c, 0)$ (which is a Euclidean Jordan algebra of rank $n-k)$, we see that the eigenvalues of $u-w$ comprise of eigenvalues of $u$ and $-w$; hence, $\lambda(u-w)$ is just a permutation of the vector formed by $\lambda(u)$ (which can be viewed as a vector in $\mathcal{R}^{k}$ ) and $-\lambda(w)$ (which can be viewed as a vector in $\mathcal{R}^{n-k}$ ). A similar statement holds for $u+w$. Hence,

$$
\|u-w\|_{q}=\|\lambda(u-w)\|_{q}=\left\|\left[\begin{array}{r}
\lambda(u) \\
-\lambda(w)
\end{array}\right]\right\|_{q}=\left\|\left[\begin{array}{c}
\lambda(u) \\
\lambda(w)
\end{array}\right]\right\|_{q}=\|\lambda(u+w)\|_{q}=\|u+w\|_{q} .
$$

Now, it it is known (see [7], page 52) that

$$
u+w \prec y .
$$

With $f(\zeta):=\|\zeta\|_{q}$ for $\zeta \in \mathcal{R}^{n}$ and $F(a):=f(\lambda(a))=\|a\|_{q}$ for $a \in \mathcal{V}$, (15) implies

$$
\|u+w\|_{q} \leq\|y\|_{q} .
$$


It follows that

$$
\|y \circ \varepsilon\|_{q}=\|u-w\|_{q}=\|u+w\|_{q} \leq\|y\|_{q}
$$

proving (81).

Now,

$$
\|x \circ y\|_{1}=\operatorname{tr}(|x \circ y|)=\langle|x \circ y|, e\rangle=\langle(x \circ y) \circ \varepsilon, e\rangle=\langle x, y \circ \varepsilon\rangle,
$$

where the last equality is due to (3). So, by (21) and (8),

$$
\|x \circ y\|_{1}=\langle x, y \circ \varepsilon\rangle \leq\langle\lambda(x), \lambda(y \circ \varepsilon)\rangle \leq\|x\|_{p}\|y \circ \varepsilon\|_{q} \leq\|x\|\left\|_{p}\right\| y \|_{q} .
$$

This completes the proof of the inequality in the theorem. Now we justify the equality statement. Suppose that $\|x \circ y\|_{1}=\|x\|_{p}\|y\|_{q}$. From (10) we have $\langle x, y \circ \varepsilon\rangle=\langle\lambda(x), \lambda(y \circ \varepsilon)\rangle$. From Theorem 1.2. we get Item $(a)$. Item $(b)$ follows from (10). Conversely, suppose conditions $(a)$ and $(b)$ hold. Then, from Theorem 1.2 and (10), $\|x \circ y\|_{1}=\langle x, y \circ \varepsilon\rangle=\|x\|\left\|_{p}\right\| y \|_{q}$.

Theorem 3.2 Let $p \in[1, \infty]$ with conjugate $q$. Then the following statements hold in $\mathcal{V}$ :

(i) $|\langle x, y\rangle| \leq\|x \circ y\|_{1} \leq\|x\|_{p}\|y\|_{q}$.

(ii) $\sup _{y \neq 0} \frac{|\langle x, y\rangle|}{\|y\|_{q}}=\sup _{y \neq 0} \frac{\|x \circ y\|_{1}}{\|y\|_{q}}=\|x\|_{p}$.

(iii) $\|x \circ y\|_{p} \leq\|x\|_{p}\|y\|_{\infty}$.

Proof. $(i)$ follows from (11) and (44). An immediate consequence of $(i)$ is:

$$
\sup _{y \neq 0} \frac{|\langle x, y\rangle|}{\|y\|_{q}} \leq \sup _{y \neq 0} \frac{\|x \circ y\|_{1}}{\|y\|_{q}} \leq\|x\|_{p}
$$

We now prove the reverse inequalities. Consider the spectral decomposition $x=\sum x_{i} e_{i}=\sum \operatorname{sgn}\left(x_{i}\right)\left|x_{i}\right| e_{i}$. First, suppose $p=\infty$. Then, for $1 \leq i \leq n$,

$$
\left|x_{i}\right|=\left|\left\langle x, e_{i}\right\rangle\right|=\frac{\left|\left\langle x, e_{i}\right\rangle\right|}{\left\|e_{i}\right\|_{1}} \leq \sup _{y \neq 0} \frac{|\langle x, y\rangle|}{\|y\|_{1}}
$$

hence $\|x\|_{\infty} \leq \sup _{y \neq 0} \frac{|\langle x, y\rangle|}{\|y\|_{1}}$. By (11), the reverse inequality also holds. Thus, (ii) holds when $p=\infty$.

Now, let $u:=\sum \operatorname{sgn}\left(x_{i}\right)\left|x_{i}\right|^{\frac{p}{q}} e_{i}$ when $1<p<\infty$ and $u:=\sum \operatorname{sgn}\left(x_{i}\right) e_{i}$ when $p=1$. We easily verify that $|\langle x, u\rangle|=\|x \circ u\|_{1}=\|x\|_{p}\|u\|_{q}$. Thus, the inequalities in (11) turn into equalities, proving $(i i)$ for $1 \leq p<\infty$.

Now,

$$
\|x \circ y\|_{p}=\sup _{z \neq 0} \frac{|\langle x \circ y, z\rangle|}{\|z\|_{q}}=\sup _{z \neq 0} \frac{|\langle x \circ z, y\rangle|}{\|z\|_{q}} \leq \sup _{z \neq 0} \frac{\|x \circ z\|_{1}\|y\|_{\infty}}{\|z\|_{q}} \leq\|x\|_{p}\|y\|_{\infty}
$$


where the first equality comes from (ii), the first inequality comes from an application of (6) , and the second inequality comes from (ii). This proves $($ iii).

Remark 1. The above result shows that the spectral norms $\|\cdot\|_{p}$ and $\|\cdot\|_{q}$ on $\mathcal{V}$ are dual to each other. We also have the following inequalities:

$$
\|x \circ y\|_{1} \leq\|x\|_{1}\|y\|_{\infty} \text { and }\|x \circ y\|_{\infty} \leq\|x\|_{\infty}\|y\|_{\infty}
$$

The first inequality has been observed in [21] in a simple algebra setting based on a case-by-case analysis.

\section{Pointwise inequalities for positive transformations}

Given $a \in \mathcal{V}$, we define the corresponding Lyapunov transformation $L_{a}$ and quadratic representation $P_{a}$ on $\mathcal{V}$ by

$$
L_{a}(x):=a \circ x \quad \text { and } \quad P_{a}(x)=2 a \circ(a \circ x)-a^{2} \circ x \quad(x \in \mathcal{V}) .
$$

Now, expressed in terms of $L_{a}$, Theorem 1.1 says that

$$
\left\|L_{a}(x)\right\|_{1} \leq\|x\|_{p}\|a\|_{q}
$$

for all $a, x \in \mathcal{V}$ and $p \in[1, \infty]$ with conjugate $q$. In this section, we consider such inequalities for quadratic representations and more generally for the so-called positive transformations. Recall that a linear transformation $P: \mathcal{V} \rightarrow \mathcal{V}$ is said to be a positive transformation [7] if

$$
x \geq 0 \Rightarrow P(x) \geq 0 .
$$

Writing $P^{*}$ for the adjoint/transpose of a linear transformation $P$, we note that if $P$ is positive, then $P^{*}$ is also positive as $\left\langle P^{*}(z), y\right\rangle=\langle z, P(y)\rangle \geq 0$ for all $y, z \geq 0$.

Here are some examples of positive transformations:

- Any nonnegative matrix on the algebra $\mathcal{R}^{n}$.

- Any quadratic representation $P_{a}$ on $\mathcal{V}[5]$.

- For any $A \in \mathcal{R}^{n \times n}$, the transformation $P$ defined on $\mathcal{S}^{n}$ by $P(X):=A X A^{T}$.

- $P=L^{-1}$ on $\mathcal{V}$, where $L: \mathcal{V} \rightarrow \mathcal{V}$ is linear, positive stable (which means that all eigenvalues of $L$ have positive real parts) and satisfies the $Z$-property [9]:

$$
x \geq 0, y \geq 0,\langle x, y\rangle=0 \Rightarrow\langle L(x), y\rangle \leq 0 .
$$

Specifically, 
(i) On the algebra $\mathcal{R}^{n}, P=A^{-1}$, where $A$ is a positive stable $Z$-matrix (meaning that its off-diagonal entries are nonpositive);

(ii) On the algebra $\mathcal{H}^{n}, P=L_{A}^{-1}$, where $A$ is a complex $n \times n$ positive stable matrix and $L_{A}(X):=A X+X A^{*}$. The transformation $L_{A}$ (also called a Lyapunov transformation) appears in dynamical systems.

- $P$ is a doubly stochastic transformation on $\mathcal{V}$ [7]. This means that $P$ is positive and $P(e)=e=P^{*}(e)$. Being a generalization of a doubly stochastic matrix, such a transformation has the following property ([7], Theorem 6):

$$
x=P(y) \Rightarrow x \prec y \text {. }
$$

- 'Schur product' induced transformation $P$ defined as follows ([11], Proposition 2.2): Fix a positive semidefinite matrix $A=\left[a_{i j}\right] \in \mathcal{S}^{n}$ and a Jordan frame $\left\{e_{1}, e_{2}, \ldots, e_{n}\right\}$ in $\mathcal{V}$. Corresponding to this Jordan frame, we write the Peirce decomposition ([5], Theorem IV.2.1) of any $x \in \mathcal{V}: x=\sum_{i \leq j} x_{i j}$. Then,

$$
P(x):=A \bullet x=\sum_{i \leq j} a_{i j} x_{i j} .
$$

It is known ([7], Example 8) that if such an $A$ has all ones on its diagonal, then $P$ is doubly stochastic.

The following result gives pointwise estimates for positive transformations.

Theorem 4.1 Let $P$ be a positive transformation on $\mathcal{V}$. For any $x \in \mathcal{V}$ and $p \in[1, \infty]$ with conjugate $q$, we have

(a) $\|P(x)\|_{1} \leq\|x\|_{p}\left\|P^{*}(e)\right\|_{q}$. In particular, $\|P(x)\|_{1} \leq\|x\|_{1}\left\|P^{*}(e)\right\|_{\infty}$.

(b) $\|P(x)\|_{p} \leq\|x\|_{\infty}\|P(e)\|_{p}$. In particular, $\|P(x)\|_{\infty} \leq\|x\|_{\infty}\|P(e)\|_{\infty}$.

Proof. (a) We start with the observation that when $u \leq v$ and $-u \leq v$ in $\mathcal{V}$, we have $\|u\|_{1} \leq\|v\|_{1}$. This is easy to see: Writing the spectral decomposition $u=\sum u_{i} e_{i}$, we have $u_{i}=\left\langle u, e_{i}\right\rangle \leq\left\langle v, e_{i}\right\rangle$ and similarly, $-u_{i} \leq\left\langle v, e_{i}\right\rangle$; thus, $\left|u_{i}\right| \leq\left\langle v, e_{i}\right\rangle$ for all $i$ and so,

$$
\|u\|_{1}=\operatorname{tr}(|u|) \leq \sum\left\langle v, e_{i}\right\rangle=\langle v, e\rangle=\operatorname{tr}(v)=\|v\|_{1}
$$

as $v \geq 0$. Now, for any $x \in \mathcal{V}, x \leq|x|$ and $-x \leq|x|$; hence using the positivity of $P, P(x) \leq P(|x|)$ and $-P(x) \leq P(|x|)$ and so

$$
\|P(x)\|_{1} \leq\|P(|x|)\|_{1}=\langle P(|x|), e\rangle=\left\langle|x|, P^{*}(e)\right\rangle \leq\left\langle\lambda(|x|), \lambda\left(P^{*}(e)\right)\right\rangle \leq\|x\|_{p}\left\|P^{*}(e)\right\|_{q}
$$

where the second inequality comes from (2) and the last inequality is just the classical Hölder's 
inequality.

(b) Since $P$ is a positive transformation, $P^{*}$ is also positive. Hence, by applying $(a)$ to $P^{*}$ and $y$, we get $\left\|P^{*}(y)\right\|_{1} \leq\|y\|_{q}\|P(e)\|_{p}$. Now, by an application of (6) , we get

$$
\|P(x)\|_{p}=\sup _{y \neq 0} \frac{|\langle P(x), y\rangle|}{\|y\|_{q}}=\sup _{y \neq 0} \frac{\left|\left\langle x, P^{*}(y)\right\rangle\right|}{\|y\|_{q}} \leq \sup _{y \neq 0} \frac{\|x\|_{\infty}\left\|P^{*}(y)\right\|_{1}}{\|y\|_{q}} \leq\|x\|_{\infty}\|P(e)\|_{p} .
$$

Here are some illustrations of the above theorem.

- Let $A \in \mathcal{R}^{n \times n}$ and consider the positive transformation $P$ on $\mathcal{S}^{n}$ defined by $P(X):=A X A^{T}$. Then, for any $X \in \mathcal{S}^{n}, P^{*}(X):=A^{T} X A$. So, with $e=I$ (the identity matrix), we have the inequalities

$$
\left\|A X A^{T}\right\|_{1} \leq\|X\|_{p}\left\|A^{T} A\right\|_{q} \text { and } \quad\left\|A X A^{T}\right\|_{p} \leq\|X\|_{\infty}\left\|A A^{T}\right\|_{p} .
$$

- Let $A=\left[a_{i j}\right] \in \mathcal{S}^{n}$ be positive semidefinite. Then, considering the 'Schur product' positive transformation $X \mapsto A \bullet X$, we have, for any $X \in \mathcal{S}^{n}$,

$$
\rho(A \bullet X)=\|A \bullet X\|_{\infty} \leq\|X\|_{\infty}\|A \bullet I\|_{\infty}=\|X\|_{\infty}\left(\max _{1 \leq i \leq n}\left|a_{i i}\right|\right)=\rho(X) \rho(\operatorname{diag}(A)),
$$

where $\rho(X)$ denotes the spectral radius of $X$ and $\operatorname{diag}(A):=A \bullet I$ with $I$ denoting the identity matrix. We remark that eigenvalue and spectral radius inequalities for the Schur/Hadamard product have been well-studied in the matrix theory literature.

We now specialize the above result to $P_{a}$. It is well-known that $P_{a}$ is self-adjoint and positive. Moreover, $P_{a}(e)=a^{2}$. Hence, we have the following: For any $a, x \in \mathcal{V}$ and $p \in[1, \infty]$ with conjugate $q$,

$$
\left\|P_{a}(x)\right\|_{1} \leq\|x\|_{p}\left\|a^{2}\right\|_{q} \text { and }\left\|P_{a}(x)\right\|_{p} \leq\|x\|_{\infty}\left\|a^{2}\right\|_{p}
$$

As we see below, some finer inequalities can be obtained.

Theorem 4.2 For any $a, x \in \mathcal{V}$,

$$
P_{a}(x) \prec a^{2} \circ x .
$$

Hence, for any $p \in[1, \infty]$ with conjugate $q$,

$$
\left\|P_{a}(x)\right\|_{1} \leq\left\|a^{2} \circ x\right\|_{1} \leq\|x\|_{p}\left\|a^{2}\right\|_{q} \quad \text { and } \quad\left\|P_{a}(x)\right\|_{p} \leq\left\|a^{2} \circ x\right\|_{p} \leq\|x\|_{\infty}\left\|a^{2}\right\|_{p} .
$$

Proof. The inequalities in (13) follow from (12) by an application of (15) (with $f$ denoting the usual $p$-norm on $\mathcal{R}^{n}$ ) and Theorem 3.2. We now prove (12). Since $P_{a}(x) \prec a^{2} \circ x$ means that $\lambda\left(P_{a}(x)\right) \prec \lambda\left(a^{2} \circ x\right)$, by continuity of the eigenvalue map $\lambda$ and the compactness of the set of 
all $n \times n$ doubly stochastic matrices, it is enough to prove (12) when $a$ is invertible (that is, all eigenvalues of $a$ are nonzero). So, assume that $a$ is invertible. Then, we have the formula (see [19], Lemma 8, Item 3)

$$
P_{a, a^{-1}} P_{a}=L_{a^{2}}
$$

where $P_{a, a^{-1}}:=L_{a} L_{a^{-1}}+L_{a^{-1}} L_{a}-L_{a \circ a^{-1}}$. By Lemma 7.1 in the Appendix, the linear transformation $P_{a, a^{-1}}$ is invertible and its inverse, $\left(P_{a, a^{-1}}\right)^{-1}$, is doubly stochastic. Now, writing

$$
P_{a}(x)=\left(P_{a, a^{-1}}\right)^{-1}\left(a^{2} \circ x\right)
$$

and invoking Theorem 6 in [7], we see that $P_{a}(x) \prec a^{2} \circ x$.

We now mention some consequences of the majorization inequality (12).

(1) Writing $\lambda_{\max }(u)$ and $\lambda_{\min }(u)$ for the maximum and minimum of eigenvalues of $u$, we have

$$
\lambda_{\max }\left(P_{a}(x)\right) \leq \lambda_{\max }\left(a^{2} \circ x\right) \quad \text { and } \quad \lambda_{\min }\left(P_{a}(x)\right) \geq \lambda_{\min }\left(a^{2} \circ x\right) .
$$

(2) When $a \geq 0$, we have $P_{\sqrt{a}}(x) \prec a \circ x$. Such a majorization inequality was proved in [17] on a case-by-case basis under the assumptions that $\mathcal{V}$ is simple, $a>0, x>0$, and $a \circ x>0$.

(3) When $a \geq 0$, for any real number $\mu, P_{\sqrt{a}}(x)-\mu e \prec a \circ x-\mu e$. It follows from (5) that for any $p \in[1, \infty]$,

$$
\left\|P_{\sqrt{a}}(x)-\mu e\right\|_{p} \leq\|a \circ x-\mu e\|_{p} .
$$

Such inequalities, for $p \in\{2, \infty\}$, appear in interior point methods, see e.g., [19], Lemma 30 .

\section{Norms of Lyapunov transformations, quadratic representations, and positive transformations}

In a recent paper [7], it was shown that for a positive transformation $P$ on a Euclidean Jordan algebra, the infinity norm of $P$ is attained at the unit element. This result applies to the quadratic transformation $P_{a}$ and the inverse of a positive stable $Z$-transformation [9] on a Euclidean Jordan algebra. In this section, we consider calculating the norms of the Lyapunov transformation $L_{a}$, the quadratic representation $P_{a}$, and positive transformations relative to spectral norms. We remark that questions related to the norm of the Lyapunov transformation $L_{A}$ on $\mathcal{S}^{n}$ (defined by $L_{A}(X):=$ $A X+X A^{T}$ for $A \in \mathcal{R}^{n \times n}$ ) arise in connection with stability of dynamical systems, see [6] and citations therein.

Given a linear transformation $T: \mathcal{V} \rightarrow \mathcal{V}$, and $r, s \in[1, \infty]$, we define the norm of the operator 
$T:\left(\mathcal{V},\|\cdot\|_{r}\right) \rightarrow\left(\mathcal{V},\|\cdot\|_{s}\right)$ by

$$
\|T\|_{r \rightarrow s}:=\sup _{x \neq 0} \frac{\|T(x)\|_{s}}{\|x\|_{r}}
$$

By the duality of norms (see Theorem $\underline{3.2}$ ), we immediately see that

$$
\|T\|_{r \rightarrow s}=\left\|T^{*}\right\|_{s^{\prime} \rightarrow r^{\prime}},
$$

where $T^{*}$ denotes the adjoint/transpose of $T$ and $r^{\prime}\left(s^{\prime}\right)$ denotes the conjugate of $r$ (respectively, of $s$ ).

Now consider the spectral decomposition $a=\sum a_{i} e_{i}$. Then,

$$
L_{a}\left(e_{i}\right)=a_{i} e_{i} \quad \text { and } \quad P_{a}\left(e_{i}\right)=a_{i}^{2} e_{i}
$$

for all $i$. For any $r, s \in[1, \infty],\left\|e_{i}\right\|_{r}=\left\|e_{i}\right\|_{s}=1$ and so, $\left|a_{i}\right|=\left\|a_{i} e_{i}\right\|_{s}=\left\|a \circ e_{i}\right\|_{s} \leq$ $\left\|L_{a}\right\|_{r \rightarrow s}\left\|e_{i}\right\|_{r}=\left\|L_{a}\right\|_{r \rightarrow s}$. Taking the maximum over $i$, we see that

$$
\|a\|_{\infty} \leq\left\|L_{a}\right\|_{r \rightarrow s} \quad(r, s \in[1, \infty]) .
$$

Similarly,

$$
\left\|a^{2}\right\|_{\infty}=\|a\|_{\infty}^{2} \leq\left\|P_{a}\right\|_{r \rightarrow s} \quad(r, s \in[1, \infty])
$$

Theorem 5.1 For any $a \in \mathcal{V}$ and $p \in[1, \infty]$ with conjugate $q$, the following statements hold:

(i) $\left\|L_{a}\right\|_{\infty \rightarrow q}=\left\|L_{a}\right\|_{p \rightarrow 1}=\|a\|_{q}$.

(ii) $\left\|L_{a}\right\|_{1 \rightarrow q}=\left\|L_{a}\right\|_{p \rightarrow \infty}=\|a\|_{\infty}$.

(iii) $\left\|L_{a}\right\|_{p \rightarrow p}=\|a\|_{\infty}$.

Proof. (i) As $L_{a}$ is self-adjoint, the first equality comes from the duality of norms. The second equality is immediate from Item $(i i)$ in Theorem 3.2 .

(ii) The first equality is due to the duality of norms. Now for the second equality. We have, from Remark 1, $\|a \circ x\|_{\infty} \leq\|a\|_{\infty}\|x\|_{\infty}$. As $\|x\|_{\infty} \leq\|x\|_{p}$, we see that $\|a \circ x\|_{\infty} \leq\|a\|_{\infty}\|x\|_{p}$ and so, $\left\|L_{a}\right\|_{p \rightarrow \infty} \leq\|a\|_{\infty}$. On the other hand, $\|a\|_{\infty} \leq\left\|L_{a}\right\|_{p \rightarrow \infty}$ from (14). This proves the equality $\left\|L_{a}\right\|_{p \rightarrow \infty}=\|a\|_{\infty}$.

(iii) From Theorem $\left[3.2(\right.$ iii $),\|a \circ x\|_{p} \leq\|x\|_{p}\|a\|_{\infty}$. From this, we get $\left\|L_{a}\right\|_{p \rightarrow p} \leq\|a\|_{\infty}$. On the other hand, $\|a\|_{\infty} \leq\left\|L_{a}\right\|_{p \rightarrow p}$ from (14). Thus, $\|a\|_{\infty}=\left\|L_{a}\right\|_{p \rightarrow p}$.

Theorem 5.2 Let $P$ be a positive transformation on $\mathcal{V}$ and $p \in[1, \infty]$ with conjugate $q$. Then,

(i) $\|P\|_{\infty \rightarrow p}=\|P(e)\|_{p}$ and $\|P\|_{p \rightarrow 1}=\left\|P^{*}(e)\right\|_{q}$. 
(ii) $\|P\|_{p \rightarrow \infty} \leq\|P(e)\|_{\infty}$ and $\|P\|_{1 \rightarrow p} \leq\left\|P^{*}(e)\right\|_{\infty}$.

Proof. (i) From Theorem 4.1(b), $\|P(x)\|_{p} \leq\|x\|_{\infty}\|P(e)\|_{p}$ with equality when $x=e$. Hence $\|P\|_{\infty \rightarrow p}=\sup _{x \neq 0} \frac{\|P(x)\|_{p}}{\|x\|_{\infty}}=\|P(e)\|_{p}$. The dual version of this gives the second statement in $(i)$. (ii) From Theorem 4.1(b), we have $\|P(x)\|_{\infty} \leq\|x\|_{\infty}\|P(e)\|_{\infty} \leq\|x\|\left\|_{p}\right\| P(e) \|_{\infty}$. This gives $\|P\|_{p \rightarrow \infty} \leq\|P(e)\|_{\infty}$. The second statement is the dual version of this.

Remark 2. The above result shows that for a positive transformation on $\mathcal{V},\|P\|_{1 \rightarrow 1} \leq\left\|P^{*}(e)\right\|_{\infty}$ and $\|P\|_{\infty \rightarrow \infty} \leq\|P(e)\|_{\infty}$. Using Theorem 6.1 (see the next section), for any $p \in[1, \infty]$ we have

$$
\|P\|_{p \rightarrow p} \leq\left\|P^{*}(e)\right\|_{\infty}^{\frac{1}{p}}\|P(e)\|_{\infty}^{1-\frac{1}{p}}
$$

and when $P$ is self-adjoint, $\|P\|_{p \rightarrow p} \leq\|P(e)\|_{\infty}$. To see a special case, suppose $L: \mathcal{V} \rightarrow \mathcal{V}$ is linear, positive stable, and satisfies the $Z$-property (see Section 3 for definitions). Then,

$$
\left\|L^{-1}\right\|_{p \rightarrow p} \leq\left\|\left(L^{*}\right)^{-1}(e)\right\|_{\infty}^{\frac{1}{p}}\left\|L^{-1}(e)\right\|_{\infty}^{1-\frac{1}{p}} .
$$

In particular, by taking $\mathcal{V}=\mathcal{H}^{n}$ and $L=L_{A}$ with $A$ positive stable (see Section 3 ), we can estimate $\left\|L_{A}^{-1}\right\|_{p \rightarrow p}$. See [2] for a discussion of this type of an estimate on the space of all $n \times n$ complex matrices.

For quadratic representations, we can compute the norms precisely.

Theorem 5.3 For any $a \in \mathcal{V}$ and $p \in[1, \infty]$ with conjugate $q$, the following statements hold:

(i) $\left\|P_{a}\right\|_{p \rightarrow 1}=\left\|P_{a}\right\|_{\infty \rightarrow q}=\left\|a^{2}\right\|_{q}$.

(ii) $\left\|P_{a}\right\|_{1 \rightarrow p}=\left\|P_{a}\right\|_{q \rightarrow \infty}=\|a\|_{\infty}^{2}$.

(iii) $\left\|P_{a}\right\|_{p \rightarrow p}=\|a\|_{\infty}^{2}$.

Proof. (i) Since $P_{a}$ is self-adjoint and $P_{a}(e)=a^{2}$, this comes from the previous theorem, Item $(i)$. (ii) From Item (ii) in the previous theorem, $\left\|P_{a}\right\|_{1 \rightarrow p}=\left\|P_{a}\right\|_{q \rightarrow \infty} \leq\|a\|_{\infty}^{2}$. The reverse inequality follows from (15).

As a consequence of Item $(i i),\left\|P_{a}\right\|_{1 \rightarrow 1}=\|a\|_{\infty}^{2}=\left\|P_{a}\right\|_{\infty \rightarrow \infty}$. By invoking Theorem 6.1 (see the next section), we see that $\left\|P_{a}\right\|_{p \rightarrow p} \leq\|a\|_{\infty}^{2}$. Since the reverse inequality also holds, see (15), we have $\left\|P_{a}\right\|_{p \rightarrow p}=\|a\|_{\infty}^{2}$.

\section{An interpolation theorem}

In this section, we prove the following interpolation theorem for a linear transformation on $\mathcal{V}$ with respect to the spectral norms. 
Theorem 6.1 Suppose $1 \leq r, s, p \leq \infty, 0 \leq \theta \leq 1$, and

$$
\frac{1}{p}=\frac{1-\theta}{r}+\frac{\theta}{s}
$$

Then, for any linear transformation $T: \mathcal{V} \rightarrow \mathcal{V}$ we have

$$
\|T\|_{p \rightarrow p} \leq\|T\|_{r \rightarrow r}^{1-\theta}\|T\|_{s \rightarrow s}^{\theta}
$$

In particular,

$$
\|T\|_{p \rightarrow p} \leq\|T\|_{1 \rightarrow 1}^{\frac{1}{p}}\|T\|_{\infty \rightarrow \infty}^{1-\frac{1}{p}}
$$

There are numerous interpolation theorems in analysis, two classical ones being the Riesz-Thorin and Marcinkiewicz interpolation theorems. The interpolation theorems are usually proved using either the real or the complex methods. We present a proof of the above theorem using the $K$-method of real interpolation theory [15]. While the above result deals with the norm of $T$ relative to the same spectral norm ( such as $\|T\|_{p \rightarrow p}$ ), we anticipate a broader result similar to the Riesz-Thorin theorem that deals with the norm of $T$ relative to two spectral norms (such as $\|T\|_{p_{0} \rightarrow p_{1}}$ ). We note that a Riesz-Thorin type result is available for linear transformations on the space of complex $n \times n$ matrices with respect to Schatten $p$-norms, see the interpolation theorem of Calderón-Lions ([18, Theorem IX.20). A key idea in our proof is the use of a majorization result that connects a $K$-functional defined on $\mathcal{V}$ to a $K$-functional on an $L_{p^{-}}$-space.

Before presenting the proof, we describe some background material. Corresponding to our Euclidean Jordan algebra $\mathcal{V}$ of rank $n$, we let $\Omega:=\{1,2, \ldots, n\}$ and $\mu$ denote the measure on (the power set of) $\Omega$ with $\mu(\{k\})=1$ for all $k \in \Omega$. Let $L_{p}(\Omega)$ (abbreviated as $L_{p}$ ) denote the corresponding Lebesgue measure space (consisting, for our consideration, only of real valued functions). We regard any element $f$ in $L_{p}(\Omega)$ either as an $n$-tuple or as a real valued function on $\Omega$. We let $\|f\|_{p}$ denote the usual $p$-norm of $f$. We assume the notation/conditions of Theorem 6.1. We will use the following abbreviations:

$$
V_{r}:=\left(V,\|\cdot\|_{r}\right), V_{s}:=\left(V,\|\cdot\|_{s}\right), M_{r}:=\|T\|_{r \rightarrow r}, \text { and } M_{s}:=\|T\|_{s \rightarrow s} .
$$

For any real number $t>0, x \in \mathcal{V}$, and $f: \Omega \rightarrow \mathcal{R}$, we consider all possible decompositions $x=a+b$ with $a, b \in \mathcal{V}$ and $f=g+h$ with $g, h: \Omega \rightarrow \mathcal{R}$, and define the $K$-functionals:

$$
K\left(t, x, V_{r}, V_{s}\right):=\inf \left\{\|a\|_{r}+t\|b\|_{s}: x=a+b\right\}
$$

and

$$
K\left(t, f, L_{r}, L_{s}\right):=\inf \left\{\|g\|_{r}+t\|h\|_{s}: f=g+h\right\} .
$$

We describe/recall some preliminary results. 
Proposition 6.2 ([15], Definition 1.2 and Example 1.27) Suppose $1 \leq r, s, p \leq \infty, r<s, 0<\theta<$ 1 , and

$$
\frac{1}{p}=\frac{1-\theta}{r}+\frac{\theta}{s}
$$

Then, for any $f: \Omega \rightarrow \mathcal{R}$,

$$
\|f\|_{p}=\left[\int_{0}^{\infty}\left(t^{-\theta} K\left(t, f, L_{r}, L_{s}\right)\right)^{p} \frac{d t}{t}\right]^{\frac{1}{p}}
$$

The following can be regarded as a majorization result. In a simple Euclidean Jordan algebra, it is known that $\lambda(a+b) \prec \lambda(a)+\lambda(b)$ [10] so that $\lambda(a+b)=A(\lambda(a)+\lambda(b))$ for some doubly stochastic matrix $A$. For a general Euclidean Jordan algebra, we have the following.

Proposition 6.3 ([13], Proposition 8) Given $a, b \in \mathcal{V}$, there exist doubly stochastic matrices $A$ and $B$ in $\mathcal{R}^{n \times n}$ such that

$$
\lambda(a+b)=A \lambda(a)+B \lambda(b)
$$

Based on the above majorization result, we connect the two $K$-functionals defined earlier.

Lemma 6.4 For any $t>0$ and $x \in \mathcal{V}$, we have

$$
K\left(t, x, V_{r}, V_{s}\right)=K\left(t, \lambda(x), L_{r}, L_{s}\right) .
$$

Proof. We fix $x \in \mathcal{V}$ and consider the decomposition $x=a+b$. By Proposition 6.3,

$$
\lambda(x)=A \lambda(a)+B \lambda(b),
$$

where $A$ and $B$ are doubly stochastic matrices in $\mathcal{R}^{n \times n}$. Let $g:=A \lambda(a)$ and $h:=B \lambda(b)$ so that

$$
\lambda(x)=g+h .
$$

As $A$ and $B$ are convex combinations of permutation matrices (by Birkhoff's Theorem [3]), we see that $\|g\|_{r} \leq\|\lambda(a)\|_{r}=\|a\|_{r}$ and $\|h\|_{s} \leq\|\lambda(b)\|_{s}=\|b\|_{s}$. Hence, for any $t>0$,

$$
K\left(t, \lambda(x), L_{r}, L_{s}\right) \leq\|g\|_{r}+t\|h\|_{s} \leq\|a\|_{r}+t\|b\|_{s} .
$$

As this holds for any decomposition $x=a+b$, taking the infimum,

$$
K\left(t, \lambda(x), L_{r}, L_{s}\right) \leq K\left(t, x, V_{r}, V_{s}\right) .
$$

Now for the reverse inequality. Consider any decomposition $\lambda(x)=g+h$ where $g, h: \Omega \rightarrow \mathcal{R}$. Corresponding to the spectral decomposition $x=\sum \lambda_{i}(x) e_{i}$, we define

$$
a:=\sum g(i) e_{i} \quad \text { and } \quad b:=\sum h(i) e_{i}
$$


Then, $x=a+b$ in $\mathcal{V}$. So,

$$
K\left(t, x, V_{r}, V_{s}\right) \leq\|a\|_{r}+t\|b\|_{s}=\|g\|_{r}+t\|h\|_{s} .
$$

Taking the infimum, we get

$$
K\left(t, x, V_{r}, V_{s}\right) \leq K\left(t, \lambda(x), L_{r}, L_{s}\right) .
$$

This completes the proof of the lemma.

Lemma 6.5 Let $T: \mathcal{V} \rightarrow \mathcal{V}$ be linear and nonzero. Then,

$$
K\left(t, T(x), V_{r}, V_{s}\right) \leq M_{r} K\left(\frac{M_{s}}{M_{r}} t, x, V_{r}, V_{s}\right) .
$$

Proof. Fix $x \in \mathcal{V}$ with decomposition $x=a+b$. Then, $T(x)=T(a)+T(b)$ and so,

$$
K\left(t, T(x), V_{r}, V_{s}\right) \leq\|T(a)\|_{r}+t\|T(b)\|_{s} \leq M_{r}\|a\|_{r}+t M_{s}\|b\|_{s} \leq M_{r}\left[\|a\|_{r}+t \frac{M_{s}}{M_{r}}\|b\|_{s}\right] .
$$

Taking the infimum over all decompositions $x=a+b$, we get the stated inequality.

We now come to the proof of Theorem 6.1.

Proof. As (17) holds when $T=0$ or $r=s$ or when $\theta \in\{0,1\}$, we assume that $T \neq 0, r \neq s$, and $0<\theta<1$. We first assume that $r<s$ (so that conditions of Proposition 6.2 are met).

We fix $x \in \mathcal{V}$ and let $y:=T(x)$. Then, $\|y\|_{p}=\|\lambda(y)\|_{p}$ and

$$
\begin{aligned}
\|\lambda(y)\|_{p}^{p} & =\int_{0}^{\infty}\left[t^{-\theta} K\left(t, \lambda(y), L_{r}, L_{s}\right)\right]^{p} \frac{d t}{t} \\
& =\int_{0}^{\infty}\left[t^{-\theta} K\left(t, y, V_{r}, V_{s}\right)\right]^{p} \frac{d t}{t} \\
& \leq \int_{0}^{\infty}\left[t^{-\theta} M_{r} K\left(\frac{M_{s}}{M_{r}} t, x, V_{r}, V_{s}\right)\right]^{p} \frac{d t}{t} \\
& =\int_{0}^{\infty}\left[\left(\frac{M_{r}}{M_{s}} t\right)^{-\theta} M_{r} K\left(t, x, V_{r}, V_{s}\right)\right]^{p} \frac{d t}{t} \\
& =\left(\frac{M_{r}}{M_{s}}\right)^{-\theta p} M_{r}^{p} \int_{0}^{\infty}\left[t^{-\theta} K\left(t, x, V_{r}, V_{s}\right)\right]^{p} \frac{d t}{t} \\
& =M_{r}^{p(1-\theta)} M_{s}^{p \theta}\|x\|_{p}^{p},
\end{aligned}
$$

where the first equality is due to Proposition 6.2, the second equality is due to Lemma 6.4, the first inequality is due to Lemma 6.5, and the third equality is due to a change of variable. Also, the last equality is seen by applying Lemma 6.4 and Proposition 6.2. Hence,

$$
\|T(x)\|_{p} \leq M_{r}^{1-\theta} M_{s}^{\theta}\|x\|_{p} .
$$

This implies that

$$
\|T\|_{p \rightarrow p} \leq\|T\|_{r \rightarrow r}^{1-\theta}\|T\|_{s \rightarrow s}^{\theta} .
$$


Note that we proved this inequality under the assumption that $r<s$. When $s<r$, we let $\phi:=1-\theta$ and observe that $\frac{1}{p}=\frac{1-\phi}{s}+\frac{\phi}{r}$. Then, by what has been proved,

$$
\|T\|_{p \rightarrow p} \leq\|T\|_{s \rightarrow s}^{1-\phi}\|T\|_{r \rightarrow r}^{\phi}=\|T\|_{r \rightarrow r}^{1-\theta}\|T\|_{s \rightarrow s}^{\theta} .
$$

We thus have (17) in all cases. In particular, by putting $r=1$ and $s=\infty$ we get

$$
\|T\|_{p \rightarrow p} \leq\|T\|_{1 \rightarrow 1}^{\frac{1}{p}}\|T\|_{\infty \rightarrow \infty}^{1-\frac{1}{p}}
$$

\section{Appendix}

For $a, b \in \mathcal{V}$, we define (see [19], page 4 or [5], page 32)

$$
P_{a, b}:=L_{a} L_{b}+L_{b} L_{a}-L_{a \circ b} .
$$

Lemma 7.1 Suppose $a \in \mathcal{V}$ is invertible. Then, the linear transformation $P_{a, a^{-1}}$ is invertible and $\left(P_{a, a^{-1}}\right)^{-1}$ is doubly stochastic.

Proof. We consider the spectral decomposition $a=\sum a_{i} e_{i}$, where the eigenvalues $a_{i}$ are nonzero and $\left\{e_{1}, e_{2}, \ldots, e_{n}\right\}$ is a Jordan frame. With respect to this Jordan frame, we consider the Peirce

decomposition of any element $u \in \mathcal{V}$ ([5], Theorem IV.2.1) in the form $u=\sum_{i \leq j} u_{i j}$. Then, from [1] (page 720),

$$
L_{a}(u)=\sum_{i \leq j} \frac{a_{i}+a_{j}}{2} u_{i j} \quad \text { and } \quad L_{a^{-1}}(u)=\sum_{i \leq j} \frac{a_{i}^{-1}+a_{j}^{-1}}{2} u_{i j} .
$$

Since $a \circ a^{-1}=e$, an easy computation shows that

$$
P_{a, a^{-1}}(u)=\sum_{i \leq j} \frac{a_{i}^{2}+a_{j}^{2}}{2 a_{i} a_{j}} u_{i j}
$$

As $P_{a, a^{-1}}(u)=0 \Rightarrow u=0$, the linear transformation $P_{a, a^{-1}}$ is invertible and

$$
\left(P_{a, a^{-1}}\right)^{-1}(u)=\sum_{i \leq j} \frac{2 a_{i} a_{j}}{a_{i}^{2}+a_{j}^{2}} u_{i j} .
$$

Now consider the real symmetric matrix $A=\left[a_{i j}\right]$, where $a_{i j}=\frac{2 a_{i} a_{j}}{a_{i}^{2}+a_{j}^{2}}$. By considering the functions $\phi_{i}(t)$ in $L_{2}([0, \infty)), i=1,2, \ldots, n$, defined by

$$
\phi_{i}(t)=\sqrt{2} a_{i} e^{-a_{i}^{2} t},
$$

we see that the inner product (computed in $L_{2}([0, \infty))$ )

$$
\left\langle\phi_{i}, \phi_{j}\right\rangle=\int_{0}^{\infty} 2 a_{i} a_{j} e^{-\left(a_{i}^{2}+a_{j}^{2}\right) t} d t=a_{i j} .
$$


Hence, $A$ is the Gram matrix corresponding to the set $\left\{\phi_{1}, \phi_{2}, \ldots, \phi_{n}\right\}$ in $L_{2}([0, \infty))$. It follows that $A$ is positive semidefinite. Now, using the definition of 'Schur product' induced transformation (see Section 4)

$$
\left(P_{a, a^{-1}}\right)^{-1}(u)=A \bullet u(u \in \mathcal{V}) .
$$

As $A$ is positive semidefinite and has ones on the diagonal, from Example 8 in [7] we see that the transformation $u \rightarrow A \bullet u$ is doubly stochastic. This proves that $\left(P_{a, a^{-1}}\right)^{-1}$ is doubly stochastic.

\section{References}

[1] M. Baes, Convexity and differentiability properties of spectral functions and spectral mappings on Euclidean Jordan algebras, Linear Alg. Appl. 422 (2007) 664-700.

[2] R. Bhatia, A note on the Lyapunov equation, Linear Alg. Appl., 259 (1997) 71-76.

[3] R. Bhatia, Matrix Analysis, Graduate Texts in Mathematics, Springer-Verlag, New York, 1997.

[4] K. Fan, On a theorem of Weyl concerning eigenvalues of linear transformations I, Proc. Nat. Acad. Sci. U.S.A., 35 (1949) 652-655.

[5] J. Faraut and A. Korányi, Analysis on Symmetric Cones, Oxford University Press, Oxford, 1994.

[6] J. Feng, J. Lam, G. Yang, and Z. Li, On a conjecture about the norm of Lyapunov mappings, Linear Alg. Appl., 465 (2015) 88-103.

[7] M.S. Gowda, Positive and doubly stochastic maps, and majorization in Euclidean Jordan algebras, Linear Alg. Appl., 528 (2017) 40-61.

[8] M.S. Gowda, R. Sznajder, and J. Tao, Some P-properties for linear transformations on Euclidean Jordan algebras, Linear Alg. Appl., 393 (2004) 203-232.

[9] M.S. Gowda and J.Tao, Z-transformations on proper and symmetric cones, Math. Program., Series B, 117 (2009) 195-222.

[10] M.S. Gowda and J. Tao, The Cauchy interlacing theorem in simple Euclidean Jordan algebras and some consequences, Linear and Multi. Alg., 59 (2011) 65-86.

[11] M.S. Gowda, J. Tao, and R. Sznajder, Complementarity properties of Peirce-diagonalizable linear transformations on Euclidean Jordan algebras, Optim. Methods Soft., 27 (2012) 719-733.

[12] R. Horn and C.R. Johnson, Topics in Matrix Analysis, Cambridge University Press, New York, 1991. 
[13] J. Jeong and M.S. Gowda, Spectral sets and functions in Euclidean Jordan algebras, Linear Alg. Appl., 518 (2017) 31-56.

[14] Y. Lim, J. Kim, and L. Faybusovich, Simultaneous diagonalization on simple Euclidean Jordan algebras and its applications, Forum Math., 15 (2003) 639-644.

[15] A. Lunardi, Interpolation Theory, Third Edition, Edizioni della Normale, Pisa, 2018.

[16] A.W. Marshall, I. Olkin, and B.C. Arnold, Inequalities: Theory of majorization and its Applications, Springer (2010).

[17] Q. Meng, J. Tao, G. Wang, and X. Chi, Some norm inequalities in Euclidean Jordan algebras, Pacific Jour. Opt., 13 (2017) 315-324.

[18] M. Reed and B. Simon, Fourier Analysis, Self-adjointness (Methods of Modern Mathematical Physics, Vol. 2), Academic Press, 1975.

[19] S.H. Schmieta and F. Alizadeh, Extension of primal-dual interior point algorithms to symmettric cones, Math. Prog. A 96 (2003) 409-438.

[20] J. Tao, L. Kong, Z. Luo, and N. Xiu, Some majorization inequalities in Euclidean Jordan algebras, Linear Alg. Appl., 161 (2014) 92-122.

[21] J. Tao and X. Yuan, A characterization of minimal elements in simple Euclidean Jordan algebras, Private communication.

[22] C.M. Theobald, An inequality for the trace of the product of two symmetric matrices, Math. Proc. Cambridge Phil. Soc., 77 (1975) 265.

[23] G. Wang, J. Tao, and L. Kong, A note on an inequality involving Jordan product in Euclidean Jordan algebras, Optim. Lett., 10 (2016) 731-736. 